\title{
Photoacoustic visualization of diatom algae
}

\author{
Cvjetinovic J. ${ }^{1 *}$, Salimon A.I. ${ }^{2}$, Novoselova M.V. ${ }^{1}$, Sapozhnikov P.V. ${ }^{3}$, \\ Kalinina O.Yu. ${ }^{4}$, Korsunsky A.M. ${ }^{2,5}$, Gorin D.A. ${ }^{1}$ \\ ${ }^{1}$ Center for Photonics and Quantum Materials, Skolkovo Institute of Science and Technology, 3 Nobelya Str., Moscow, 121205, Russia \\ ${ }^{2}$ Center for Energy Science and Technology, Skolkovo Institute of Science and Technology, 3 Nobelya Str., Moscow, 121205, Russia \\ ${ }^{3}$ Shirshov Institute of Oceanology of Russian Academy of Sciences, 36 Nakhimovsky Prospekt, Moscow, 117997, Russia \\ ${ }^{4}$ Faculty of Geography, Lomonosov Moscow State University, 1 Leninskiye Gory, Moscow, 119991, Russia \\ ${ }^{5}$ Department of Engineering Science, University of Oxford, Oxford, OX1 3PJ, United Kingdom
}

\begin{abstract}
Photoacoustic imaging is a rapidly maturing imaging modality in biological research and medicine. Its underlying principle is the photoacoustic effect. This method can also be used to study algae containing light-absorbing chromophores, which produce a photoacoustic signal under laser irradiation. Here, we demonstrate successful photoacoustic visualization of unicellular diatom algae called Karayevia amoena. These hierarchical micro/nano-structured organisms mixed with an agarose gel were excited by nanosecond pulsed green, $532 \mathrm{~nm}$ laser using a raster scanning optoacoustic mesoscopy (RSOM) approach. We observed a strong photoacoustic signal from diatom cells and showed that it decreases with decreasing diatom concentration. The signal originates from chlorophyll $a$ and $c$ and fucoxanthin. We also studied cyanobacteria to prove that the photoacoustic technique can be useful for visualizing this type of object as well. Photoacoustic imaging is a promising method for remote control of algae cultivation in bioreactors and their natural environment.
\end{abstract}

Keywords: Diatoms, algae, cyanobacteria, photoacoustic imaging, photoacoustics

\section{Introduction}

Photoacoustic imaging is based on the absorption of light pulses and the ultrasonic emission that is generated as a result of the thermoelastic expansion of an object (Wang and $\mathrm{Hu}, 2012$ ). The acoustic response can be detected by an ultrasound transducer and reconstructed to form a photoacoustic image. We decided to use this method to study unicellular diatom algae, covered with a silica cell wall called a frustule (Korsunsky et al., 2019). Diatoms represent attractive materials for a variety of applications due to their unique structure and properties (Dolatabadi and de la Guardia, 2011; Mishra et al., 2017). Our first successful results proved that photoacoustic imaging of diatoms is feasible (Cvjetinovic et al., 2020), and inspired us to use this method to assess the activity and presence of living diatom cells, as well as to study other photosynthetic organisms such as cyanobacteria. Here we present new results that demonstrate strong photoacoustic signals from both diatoms and cyanobacteria and therefore confirm the presence of living cells within the studied probe.

\section{Materials and methods}

Karayevia amoena ( $K$. amoena) diatoms were collected from the tidal zone of Gdansk Bay (Baltic Sea, salinity $5 \mathrm{ppt}$ ) and cultivated in a 0.5 -L polyethylene terephthalate bottle on the windowsill of the laboratory at room temperature and alternating day/night regime. Colonies of cyanobacteria were grown at the surface of agar carrier from the probes collected at the coast of the Aral Sea.

For photoacoustic measurements, different $K$. amoena concentrations $(305,152.5,76.3,38.1,19.1$, 9.5, 4.8, and 2.4 cells $/ \mu \mathrm{l}$ ) were mixed with agarose and placed in the imaging chamber of RSOM Explorer P50 setup (iThera Medical, Germany). The photoacoustic signal was excited by frequency-doubled Nd:YAG laser at an excitation wavelength of $532 \mathrm{~nm}$. The samples were scanned over an $11 \times 11 \times 2.5 \mathrm{~mm}$ field of view, and the signals were detected with the LiNbO3 detector. Photoacoustic imaging of cyanobacteria colonies grown on the agar substrate was performed in the same way. 


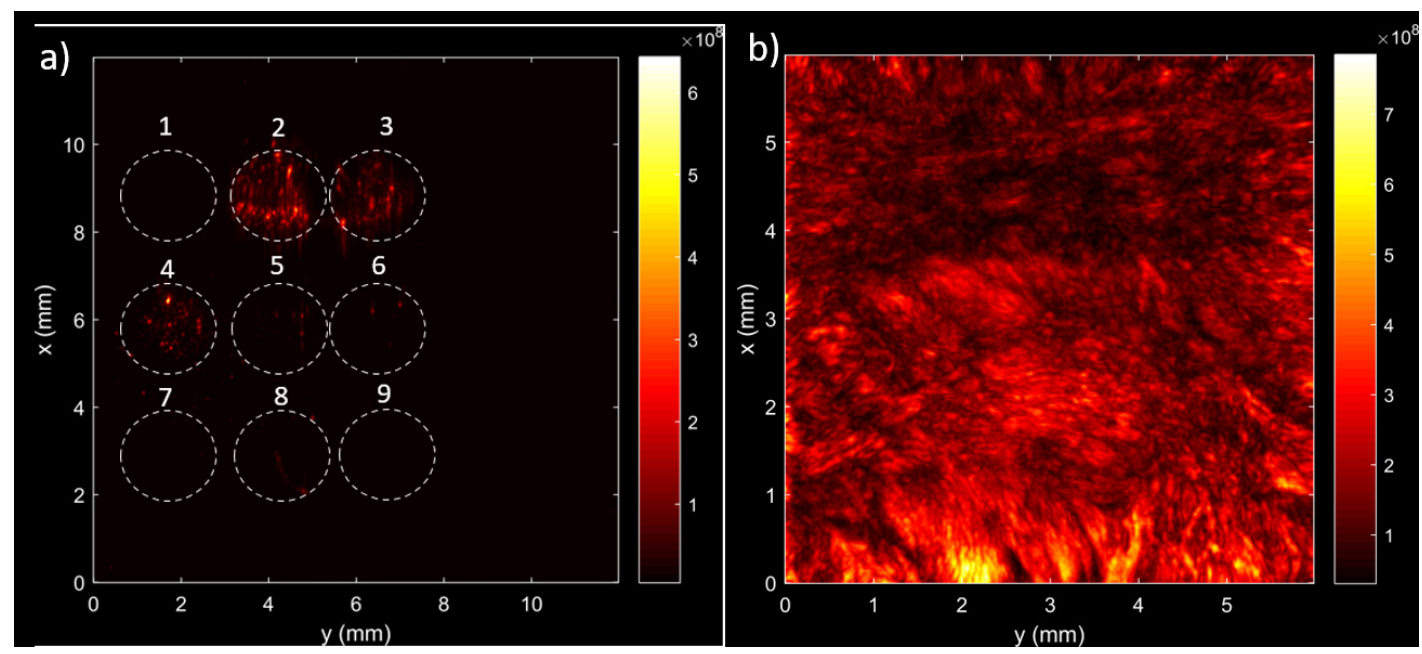

Fig. RSOM images of: A different concentrations of $K$. amoena diatoms: 1 - Agarose gel; 2 - Stock suspension; Diluted suspensions: $3-1: 2 ; 4-1: 4 ; 5-1: 8 ; 6-1: 16 ; 7-1: 32 ; 8-1: 64 ; 9-1: 128$, B cyanobacteria colonies on the agar substrate

\section{Results and discussion}

The obtained results of photoacoustic measurements of diatoms and cyanobacteria are shown in figure. RSOM images demonstrate a strong photoacoustic response from both samples.

The obtained images of diatoms were analyzed with ImageJ software, and it was shown that the signal decreases with decreasing concentration. More diluted diatom suspensions were not visible due to insufficient concentration and small cell size. According to our previous study, chlorophyll $a$ and $c$ and carotenoid fucoxanthin are responsible for the strong photoacoustic effect because the absorption value for diatoms at 532 $\mathrm{nm}$ corresponds to the obtained signal (Cvjetinovic et al., 2020). Cyanobacteria, among other pigments, also contain chlorophyll $a$. We proved that photoacoustic imaging could be easily and rapidly performed without complicated sample preparations.

\section{Conclusions}

Our research demonstrates the successful application of photoacoustic imaging of diatoms and cyanobacteria, which contain significant amounts of chromophores that provide a photoacoustic effect. We believe that the photoacoustic technique can be readily used for monitoring the activity of algae not only in laboratory conditions but also in aquatic environments.

\section{Acknowledgments}

The cultivation of $K$. Amoena diatoms was partially funded by RFBR grant no. 18-44-920012. Alexander M. Korsunsky acknowledges support from the Royal Society of London through grant no. IEC/R2/170223.

\section{References}

Cvjetinovic J., Salimon A.I., Novoselova M.V. et al. 2020. Photoacoustic and fluorescence lifetime imaging of diatoms. Photoacoustics 18: 1-9. DOI: 10.1016/j.pacs.2020.100171

Dolatabadi J.E.N., de la Guardia M. 2011. Applications of diatoms and silica nanotechnology in biosensing, drug and gene delivery, and formation of complex metal nanostructures. TrAC - Trends in Analytical Chemistry 30: 1538-1548. DOI: 10.1016/j.trac.2011.04.015

Korsunsky A.M., Sapozhnikov P.V., Salimon A.I. 2019. Nature's neat nanostructuration: the fascinating frustules of diatom algae. Materials Today 22: 159-160. DOI: 10.1016/j. mattod.2019.01.002

Mishra M., Arukha A.P., Bashir T. et al. 2017. All new faces of diatoms: potential source of nanomaterials and beyond. Frontiers in Microbiology 8: 1-8. DOI: 10.3389/ fmicb.2017.01239

Wang L.V., Hu S. 2012. Photoacoustic tomography: in vivo imaging from organelles to organs. Science 335: 14581462. DOI: $10.1126 /$ science. 1216210 